\title{
Survey to healthcare professionals on the practicality of AI
}

\section{services for healthcare [version 1; peer review: 1 approved}

\section{with reservations, 2 not approved]}

\author{
Antti Väänänen (iD, Keijo Haataja, Pekka Toivanen
}

School of Computing, University of Eastern Finland, Kuopio, Finland

\begin{tabular}{l}
\hline V1 First published: 22 Jul 2020, 9:760 \\
https://doi.org/10.12688/f1000research.23883.1 \\
Latest published: 22 Jul 2020, 9:760 \\
https://doi.org/10.12688/f1000research.23883.1 \\
\hline
\end{tabular}

\section{Abstract}

Background: Artificial Intelligence (AI) is already in use in many fields of healthcare, and utilization of AI in state-of-the art healthcare services is growing, with many studies showing that AI can provide benefits in healthcare processes. However, there is a need for research about healthcare professionals' willingness to use AI-based services, and their consideration about in which cases AI could provide better healthcare outcomes and reduce healthcare professionals' workload.

Methods: We studied the latest technologies and methods from current healthcare AI services' end-users to create a survey on healthcare services, including use cases that could be beneficial for healthcare professionals. We focused on studying Information and Communication Technology (ICT) applications and services which utilize modern AI features. The purpose of our survey for healthcare professionals was to provide an analysis of the healthcare services that utilize AI features, which could provide better health outcomes from a healthcare professional and care process point of view.

Results: AI features, such as health monitoring, medication management, and connected machines are considered to have high benefit in healthcare workflow. Moreover, we found that the majority $(67.8 \%)$ of healthcare professionals are willing to use AI for supporting decision making or even providing independent diagnosis. In total, $28.9 \%$ of healthcare professionals are willing to use AI in a restricted manner to help professionals in care processes. Only 3.3\% of respondents are not willing to use AI-based services at all.

Conclusions: Our survey indicated that the willingness of healthcare professionals to use different AI in healthcare technology solutions is high.

\section{Keywords}

Artificial Intelligence, Digital Health, Healthcare Information Systems, Automated Diagnoses.

\section{Open Peer Review \\ Approval Status ? X X \\ 12 \\ 23

version 1 ? $\times$

22 Jul 2020 view view view \\ 1. Parag Chatterjee (D), National Technological University, Buenos Aires, Argentina Universidad de la República, Montevideo, Uruguay \\ 2. Charlotte Blease, Beth Israel Deaconess Medical Center, Harvard Medical School, Boston, USA \\ 3. Lorenzo Faggioni (D), University of Pisa, Pisa, Italy} Any reports and responses or comments on the article can be found at the end of the article. 
This article is included in the Artificial

Intelligence and Machine Learning gateway.

Corresponding author: Antti Väänänen (antti.vaananen@uef.fi)

Author roles: Väänänen A: Conceptualization, Formal Analysis, Investigation, Resources, Visualization, Writing - Original Draft Preparation; Haataja K: Writing - Review \& Editing; Toivanen P: Project Administration, Supervision, Writing - Review \& Editing

Competing interests: No competing interests were disclosed.

Grant information: This study was supported by University of Eastern Finland.

Copyright: ( $\odot 2020$ Väänänen A et al. This is an open access article distributed under the terms of the Creative Commons Attribution License, which permits unrestricted use, distribution, and reproduction in any medium, provided the original work is properly cited.

How to cite this article: Väänänen $A$, Haataja $K$ and Toivanen P. Survey to healthcare professionals on the practicality of AI services for healthcare [version 1; peer review: 1 approved with reservations, 2 not approved] F1000Research 2020, 9:760 https://doi.org/10.12688/f1000research.23883.1

First published: 22 Jul 2020, 9:760 https://doi.org/10.12688/f1000research.23883.1 


\section{Introduction}

The development of Artificial Intelligence (AI) has been very fast in the past decade. AI and robotics are now utilized in almost all business sectors and this will impact the global labor market in the near future ${ }^{1}$. The following are AI services that have gained the most publicity: robotics, autonomous cars, computer vision applications, and natural language processing services. However, $\mathrm{AI}$ is already in use or is emerging on a wide scale in different business sectors ${ }^{2}$.

When considering new models for AI utilization in healthcare processes, it is generally believed that soon AI will automatically give accurate diagnoses, provide and invent new care instructions and new treatments for diseases, and will act as human healthcare professionals but in a much more accurate, precise, and effective way. In this scenario, AI could provide a remarkable advantage to enhance health outcomes and healthcare processes, as well as help caregivers in their demanding work. For example, AI can provide help regarding the shortage of the healthcare workforce and can also provide healthcare resources for aging populations ${ }^{3}$. In addition, in situations when the global healthcare system is burdened, such as the COVID-19 pandemic, healthcare resources have an increased workload and there is strong demand for AI solutions, which will provide governments and healthcare organizations with the necessary tools to perform actions faster and more efficiently, especially in pandemics ${ }^{4}$. These are the final goals of utilizing AI in healthcare, but all of these are currently not achievable at the time of writing this article.

To achieve these goals, we need to study healthcare professionals' viewpoints concerning current utilization of $\mathrm{AI}$ in healthcare and ask professional end-users (clinicians and administrators) of healthcare services about the best use cases where AI could benefit different healthcare processes. Moreover, before we can take full advantage of AI in healthcare, we must tackle ethical and legal obstacles and comply with all regulatory requirements ${ }^{5}$. Furthermore, metrics to measure benefits and better care outcomes for patients, better care outcomes for healthcare service providers, effectiveness of service, and response time for service queries, must be constructed.

In this study, we provide the results of a survey conducted to healthcare professionals about use of AI in healthcare practices. Based on the results we provide some evaluation of AI features that could be widely accepted by healthcare professionals. Moreover, we present new ideas that will be used in our future research work and insights for a novel upcoming healthcare AI-service platform. We hope that this novel AI-based healthcare solutions platform will be able to support healthcare service providers, caregivers, and customers in a more effective way compared with traditional healthcare IT services.

\section{Methods}

The aim of the survey was to evaluate utilization and acceptance of different AI-based services of healthcare professionals working in the healthcare sector. We also provide a comparative analysis of our survey and an earlier survey ${ }^{6}$, where the adoption of AI services by healthcare professionals and public users were studied.

\section{Al services in the healthcare domain}

The main areas of AI-based healthcare are clinical decision making, healthcare interventions, automatic care or healthcare process recommendations, patient administration, and patient monitoring. For this study, we searched the literature for research and utilization of AI technology in the healthcare sector. Based on our search, we selected healthcare services that included AI technologies in natural language processing, neural networks and deep learning, computer vision or robotics, rule-based expert systems, and physical robots ${ }^{7,8}$. This selection was made on the basis that there are many studies concerning these AI technologies and they are commonly in use in the healthcare sector. Therefore, healthcare services utilizing AI that were used as examples in our survey are as follows: robot-assisted surgery, virtual assistants for nursing and consultation, assistant for administration and workflow, fraud detection, dosage error reduction, connected machines, clinical trial participation, preliminary diagnosis, image diagnosis, cybersecurity, medication management, health monitoring, and drug creation.

\section{Survey design and participants}

The survey was designed to analyze the most suitable services and features to support healthcare professionals and administrators in their work, as well as which features and services could provide best care outcomes for the patients. The survey contained three parts: part 1 asked for demographic characteristics of respondents; part 2 asked general questions concerning technology and AI; and part 3 asked about an individual's acceptance of AI technology and its advantages for certain use cases. Question types were single selection, multi-selection, open number value, and 5-step Likert Scale ${ }^{9}$. The survey can be found in full in the Extended data.

The survey was created using Google Forms. A link to the survey was sent to healthcare professionals by informing healthcare professionals in our own network in Finland via Facebook and using SurveyCircle scientific survey service ${ }^{10}$. No individual requests to complete the survey were sent. The survey was accessible from December 19, 2019 to February 25, 2020.

The survey was designed to provide data in a formal structured manner to support our analysis in an optimal way and enable us to smoothly continue with our practical implementation efforts of future research work ideas.

\section{Data analysis}

We used basic statistical analysis tools from Excel Analysis ToolPak ${ }^{11}$, presenting mean values and standard deviation for each question, and analyzed respondents' staff group, work experience, and age against AI technology adoption. We analyzed how respondents utilize IT technology in their work, as well as their willingness to adopt new healthcare services that use AI technology. Finally, we compared our survey results to the survey results conducted by YouGov Research in November $2016^{6}$ to 
the general public in 12 countries across Europe, the Middle East, and Africa $(n=12000)$. Our aim was to find out if our survey results correlate with the survey results from YouGov Research.

\section{Ethical considerations}

Ethical approval for the study protocol and survey was waived by the University of Eastern Finland Research Ethics Committee. This waiver of approval was provided since the study did not contain any of the following: research deviates from principle of informed consent; research involves intervening in the physical integrity of participants; research is on minors under the age of 15 years; research exposes participants to strong stimuli; research involves risk of causing mental harm to participants; and research involves threat of safety to participants.

Participants were informed about the aim of the questionnaire and that this information would be used in research, which was shown at the beginning of the questionnaire. Completion of the questionnaire was taken as consent to participate.

\section{Results}

We created an Internet survey to examine the usage, usability, and end-user acceptance of AI-based healthcare services by healthcare professionals. We received a total of 125 responses. Of these, only 121 responses were $100 \%$ complete and therefore only these responses were analyzed.
The study population gender distribution was female $63.6 \%$ (77), male $35.6 \%$ (43), and other $0.8 \%$ (1). Mean age and mean work experience was not able to be calculated accurately because of the age and experience questions did not contain exact age or years of experience. Respondents staff group distribution was as follows: nursing staff, $48.8 \%$ (59); office and administration staff, $14.9 \%$ (18); research staff, $14.0 \%$ (17); physicians, $14 \%$ (17); social work, $5.0 \%$ (6); and maintenance staff, $3.3 \%$ (4). The demographics of the study population are shown in Figure 1.

The survey measured healthcare professionals' opinions about how AI technology will be able to provide enhancement to care processes and functions, and if AI can replace some activities or tasks that are traditionally done by healthcare professionals or traditional healthcare ICT services. The survey results were analyzed by each question and with basic statistical analysis tools (mean values and standard deviation).

Current use of ICT technology by healthcare professionals In the survey we asked about the current use of different digital services and features among healthcare professionals (question G1). When analyzing the results, we noticed that respondents stated that the most currently used ICT services listed in the survey were for drug discovery $(31.4 \%)$, medical data security (28.1\%), medical diagnosis (24\%), signal analysis $(23.1 \%)$, and finding participants to take part in clinical trials $(23.1 \%)$. The least used services were robot surgery $(6.6 \%)$ and fraud detection

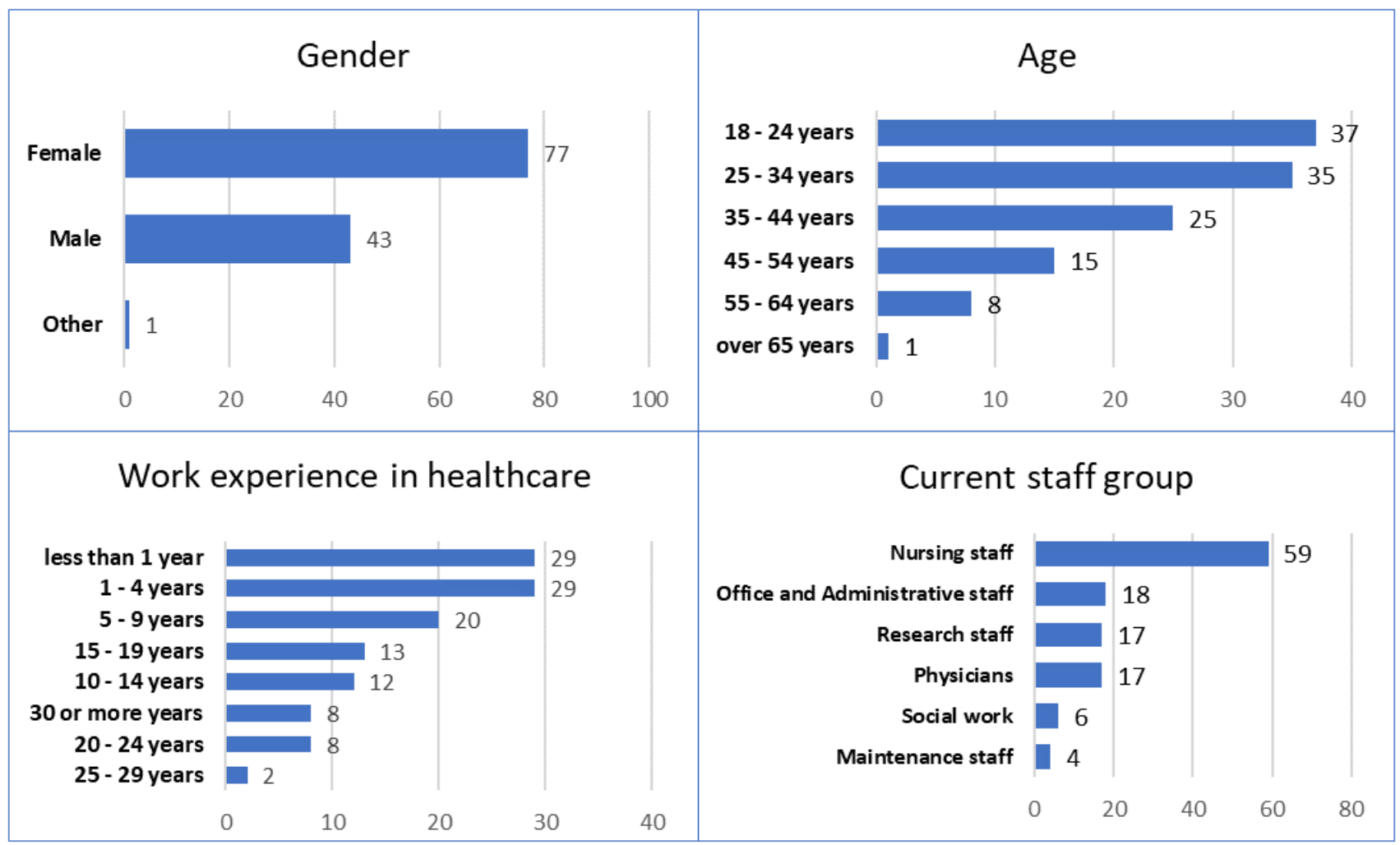

Figure 1. Participants' demographic information. 
(9.1\%). In total, $19 \%$ of professionals did not use any of the listed digital services in their work (Figure 2).

For the utilization of these ICT services, 19\% of respondents did not use any of the services listed in G1. For the technology usage level (question G3), we found that $20 \%$ of the respondents used these services daily and $24 \%$ at least once per week (Figure 3).

We also asked health professionals which healthcare ICT services and features were considered the most beneficial in their work and/or care processes (question G2). There was the possibility to select multiple technologies. The respondents reported that the following were the most beneficial/used: health monitoring $(43.8 \%)$, medication management $(32.2 \%)$, connected machines $(27.3 \%)$, dosage error reduction $(23.1 \%)$, and signal analysis (18.2\%). The least beneficial services for healthcare professionals were cybersecurity $(8.3 \%)$ and fraud detection $(9.1 \%)$. In total, $10.7 \%$ of respondents do not want to use any of the listed services in their work (Figure 4).

\section{Al advantages/disadvantages as considered by healthcare professionals}

One of the questions in the survey was for healthcare professionals to consider the advantages of AI-based services (question G4; Figure 5). The response was a 4-step Likert scale from 0 to 4 , where 0 is "Do not agree at all" and 4 is "I fully agree". The results showed that three most considered advantages of AI by healthcare professionals were "Simple and repeating tasks can be performed by AI/robots" (mean score 3.0), "AI can provide help in healthcare professionals workload" (mean score 3.0), and "AI can provide easier access for more people/patients to healthcare services" (mean score 3.0). These services have also lowest standard deviation $(0.86$ - 0.95). Other lesser considered advantages were "AI can give better treatment recommendations" (mean score 2.3) and "AI can perform surgery more accurately than healthcare professionals" (mean score 2.3). When analyzing the average scores from all replies there is an indication that healthcare professionals mostly consider AI-based services and features in healthcare to have advantages.

We also asked what disadvantages healthcare professionals consider AI to have when used in healthcare (question G5, Likert scale as for question G4; Figure 6). The highest considered disadvantage was given for "AI or robots cannot be in contact with patients. Patients need human interaction when providing healthcare services." (mean score 2.8), "Too complicated for healthcare professionals to access AI technology solutions" (mean score 2.1) and "AI does not provide accurate results for decision making. Only healthcare professionals can make right decisions." (mean score 2.1) The lowest value was given to "I can see only disadvantages when using AI or robotics in healthcare." (mean score 1.2). The results of this question show that some healthcare professionals express their concern that AI should not have contact with patients without human interaction and the final decision in healthcare processes should be kept in human hands. We can also see that healthcare professionals consider that AI-based solutions bring advantages rather than disadvantages.

Willingness to use Al services by healthcare professionals We asked healthcare professionals about willingness to use healthcare services that utilize AI (questions A1-A15). Respondents

\section{Technology already used by healthcare professionals}

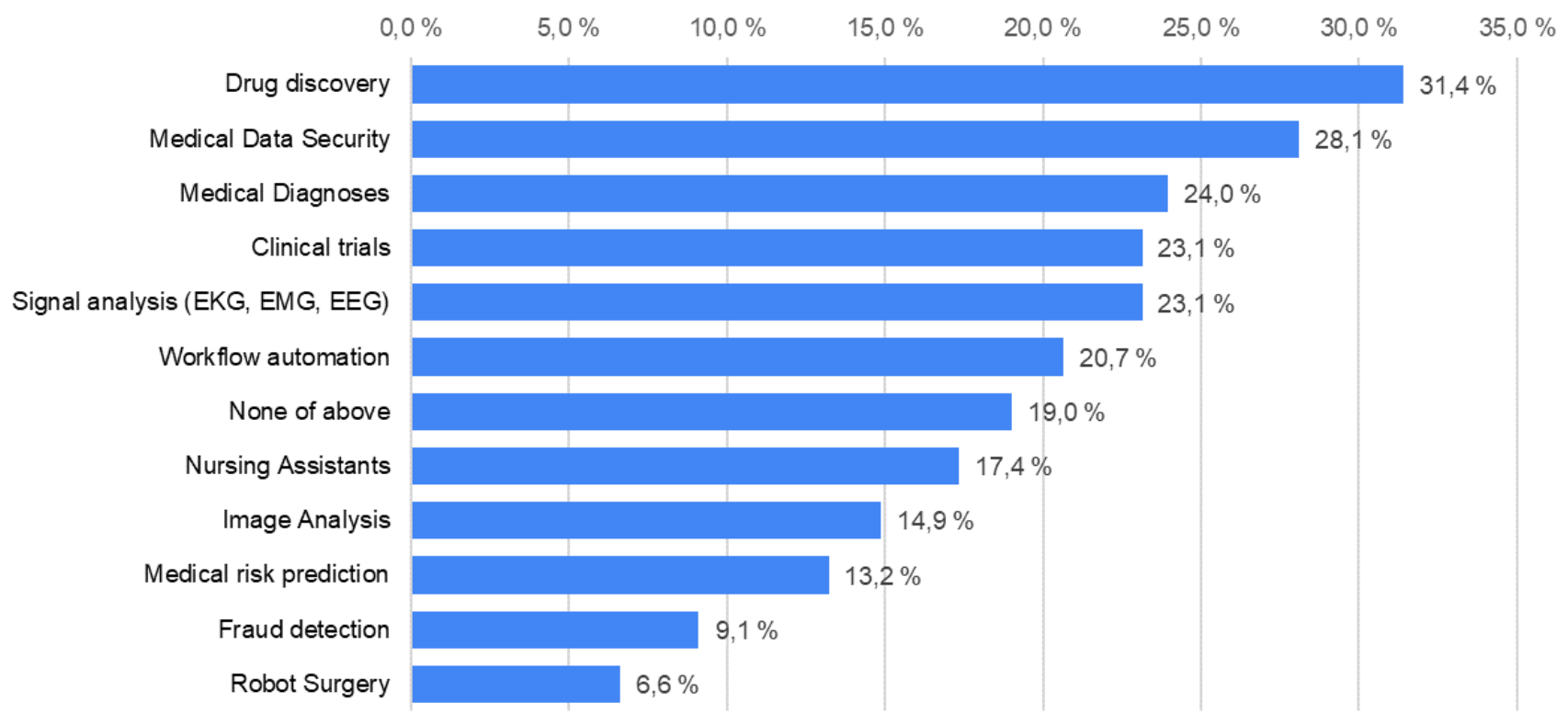

Figure 2. ICT technology currently used by respondents (question G1). 


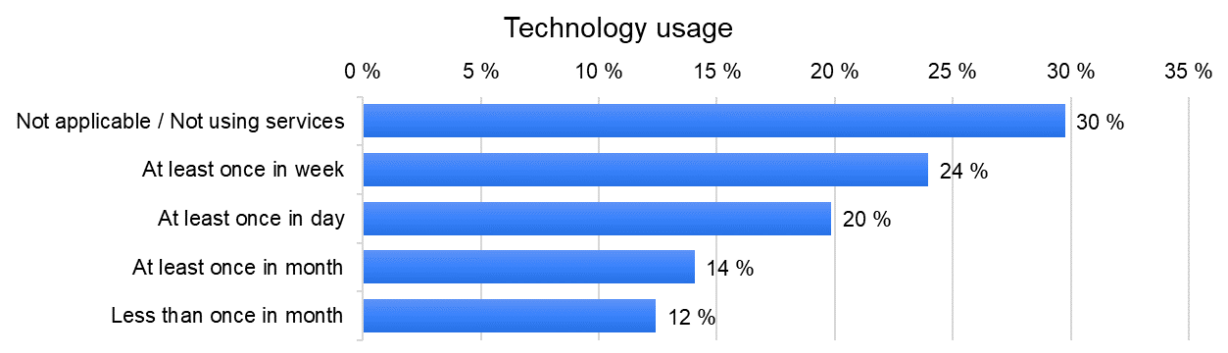

Figure 3. ICT healthcare service usage level among respondents (question G3).

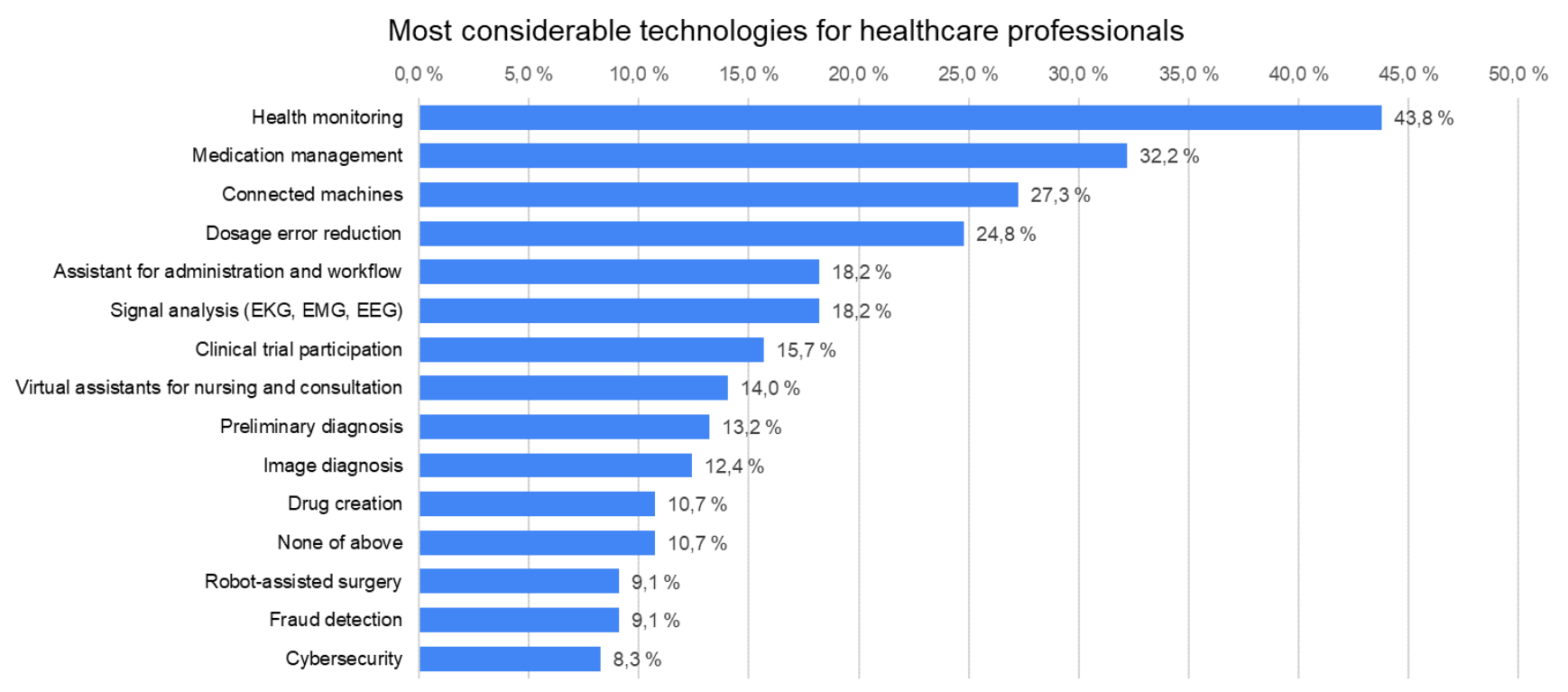

Figure 4. The most beneficial technologies as reported by healthcare professionals (question G2).

\section{Considered advantages of Al services}

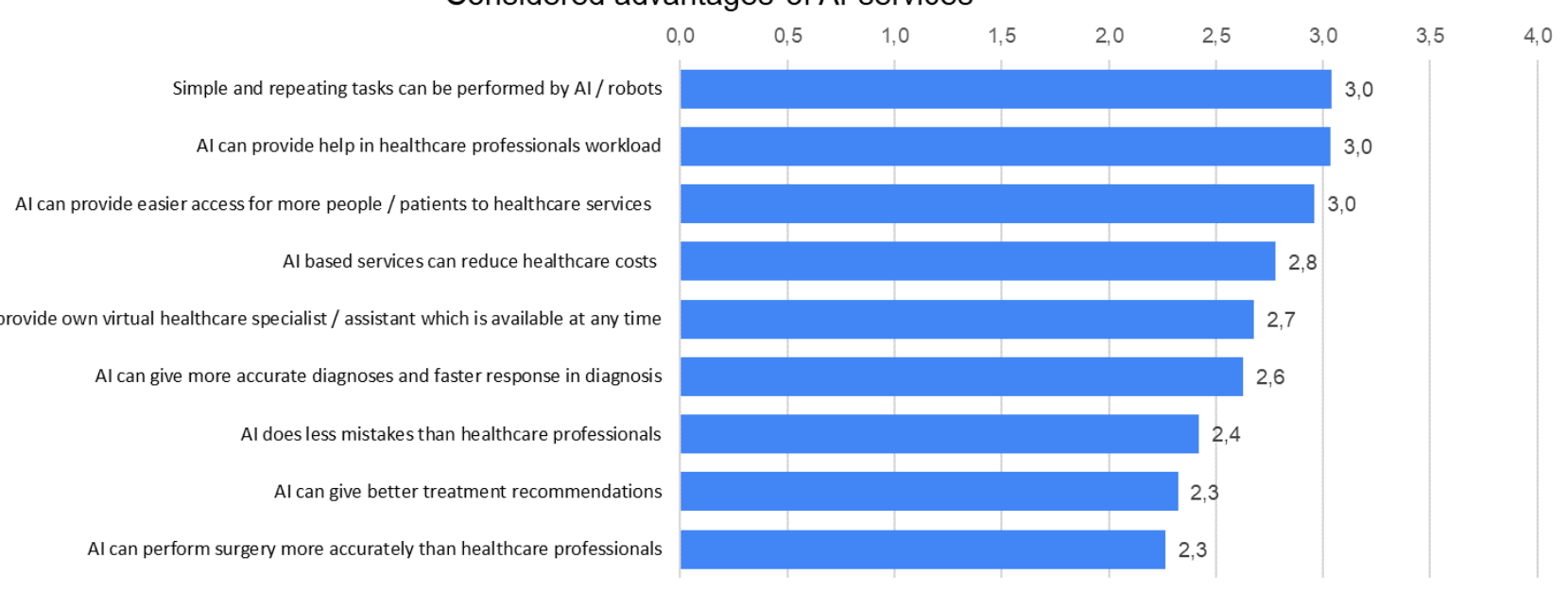

Figure 5. Advantages of Al in healthcare considered by healthcare professionals (question G4). Likert scale from 0 to 4 , where 0 is "Do not agree at all" and 4 is "I fully agree". 


\section{Disadvantages of using $\mathrm{Al}$ in healthcare}

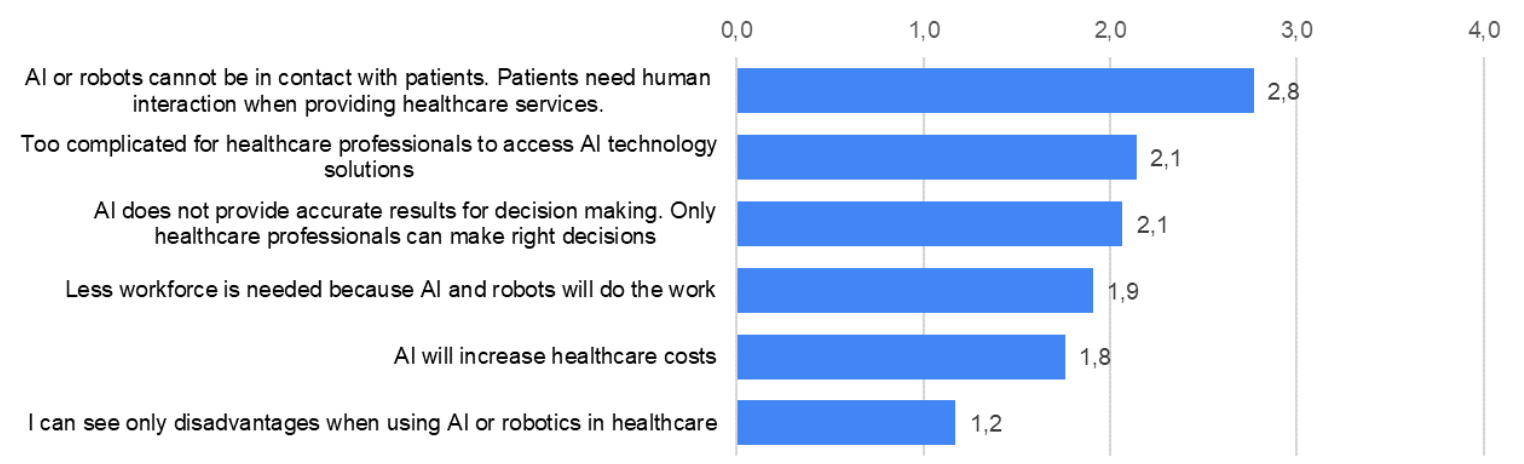

Figure 6. Disadvantages of $\mathbf{A l}$ in healthcare considered by healthcare professionals (question G5). Likert scale from 0 to 4 , where 0 is "Do not agree at all" and 4 is "I fully agree".

could evaluate each service independently with a 4-step Likert scale between 0 to 4 , with 0 as "Not willing" and 4 as "Very willing". A mean utilization score of 2.8 was given to services used for automatic health monitoring, providing enhanced data security, providing fraud detection, providing medical risk prediction, and conducting health assessment questionnaires. These services also have the lowest standard deviation $(0.80-0.90)$. Lowest utilization scores were given to services such as AI-assisted major surgery (mean score 1.9), AI giving diagnosis of patient (2.3), and AI providing instant healthcare advice for the patient (2.3). Overall results are shown in Figure 7.

Finally, we asked healthcare professionals how they are to have AI make decisions or give recommendations in healthcare processes (question G6). The responses showed that most healthcare professionals are willing to use AI in healthcare in all possible cases with the final decision made by humans $(65 \%)$ or AI to give decisions without human interaction (3\%). Also $29 \%$ of respondents consider that AI can ease healthcare work and processes, but only by giving recommendations. Only 3\% of respondents consider that $\mathrm{AI}$ is not suitable for healthcare.

\section{Correlation of responses with healthcare professionals' demographics}

We analyzed how respondents' staff group and age group correlate to the willingness to use AI technology. Social work and maintenance staff had a total 10 respondents each and were therefore combined. We found that there was only minor deviation among different staff groups supporting AI utilization (Figure 8).

Secondly, we analyzed age groups which have over 15 respondents. Age groups (55-64 years and over 65 years) had total 9 respondents and those were combined. We found that there was only minor deviation among different age groups supporting AI utilization (Figure 9).
Thirdly, we analyzed work experience from the groups that have over 15 respondents. Work experience groups (55- 64 years and over 65 years) had total 9 respondents and those are combined to last pie chart. We found that there was only minor deviation among different work experience groups supporting AI utilization (Figure 10).

\section{Discussion}

From the survey results, we found out that the AI technology that respondents consider to be most useful for healthcare work processes (question G2) are services that provide health monitoring, medication management, and networked devices and databases which can exchange data between each other. In addition, the three most currently used healthcare IT services by respondents are drug discovery, medical data security, and medical diagnosis (question G1).

We compared our research results to an earlier survey conducted by YouGov Research ${ }^{6}$. We found similarities in perceived advantages of AI between the two surveys. In the YouGov survey, the top two advantages that respondents reported were "Healthcare would be easier and quicker for more people to access" and "Advanced computers/robots can make diagnosis faster and more accurately". From our survey, the top three perceived advantages identified by healthcare professionals were "AI can provide easier access for more people/patients to healthcare services", "AI can provide help in healthcare professionals workload", and "Simple and repeating tasks can be performed by AI/robots".

When we compared disadvantages between surveys, we found that the top three disadvantages reported in our survey were "AI or robots cannot be in contact with patients. Patients need human interaction when providing healthcare services", "Too complicated for healthcare professionals to access AI technology 


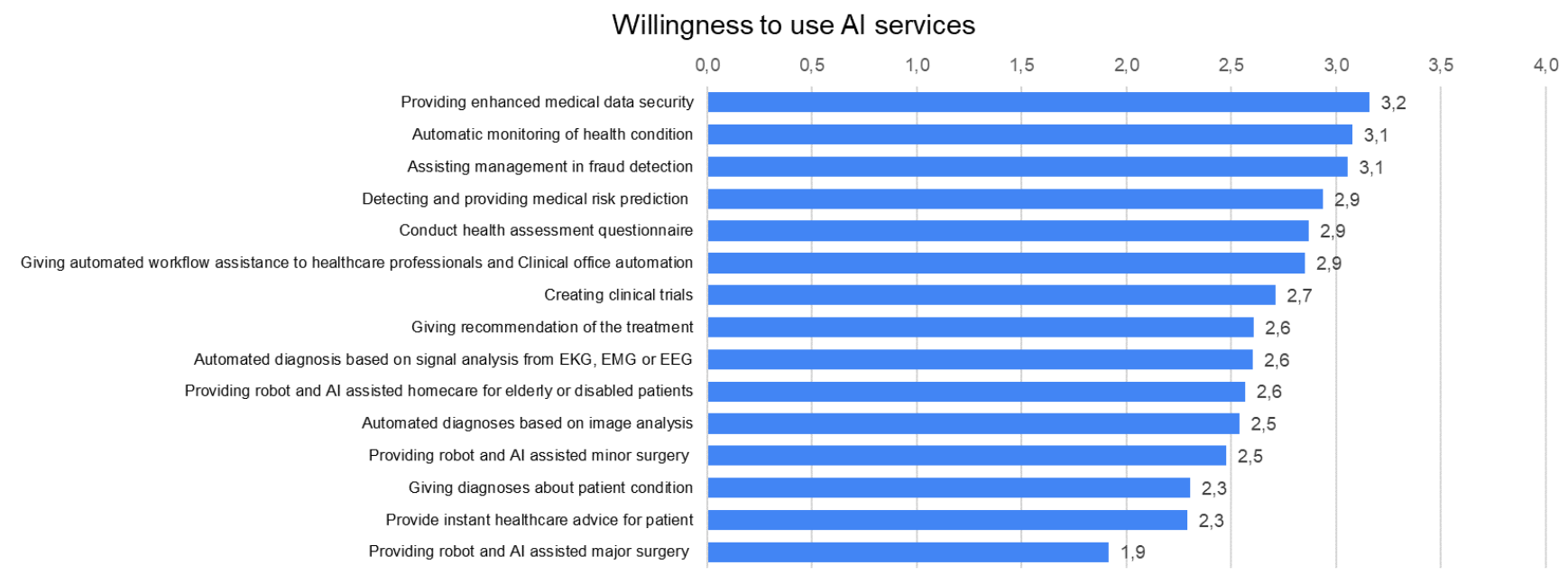

Figure 7. Willingness to use Al-based services as reported by healthcare professionals (question A1-A15). Likert scale between 0 to 4 , with 0 as "Not willing" and 4 as "Very willing".

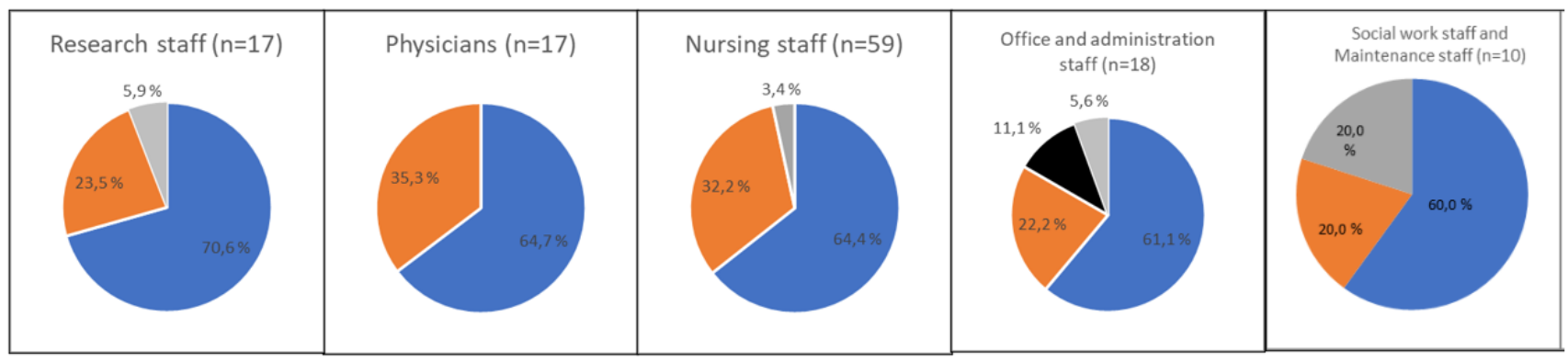

Figure 8. Reported Al utilization by healthcare professionals according to staff groups. Blue, "Al can be used in all possible cases, but humans should always make final decision"; orange, "Al can ease clinical work and clinical processes but only with restricted matter (only by giving recommendations)"; black, "Al is not suitable for healthcare"; grey, "Al can be used in healthcare and can independently provide diagnoses and decisions for care".

\begin{tabular}{|l|c|c|c|c|}
\hline Age 18 - 24 years $(n=37)$ & Age 25 - 34 years $(n=35)$ & Age $35-44$ years $(n=25)$ & Age 45 - 54 years $(n=15)$ & Age 55 and over $(n=9)$ \\
\hline $21,6 \%$ & &
\end{tabular}

Figure 9. Reported Al utilization by healthcare professionals according to age groups. Blue, "Al can be used in all possible cases, but humans should always make final decision"; orange, "Al can ease clinical work and clinical processes but only with restricted matter (only by giving recommendations)"; black, "Al is not suitable for healthcare"; grey, "Al can be used in healthcare and can independently provide diagnoses and decisions for care". 


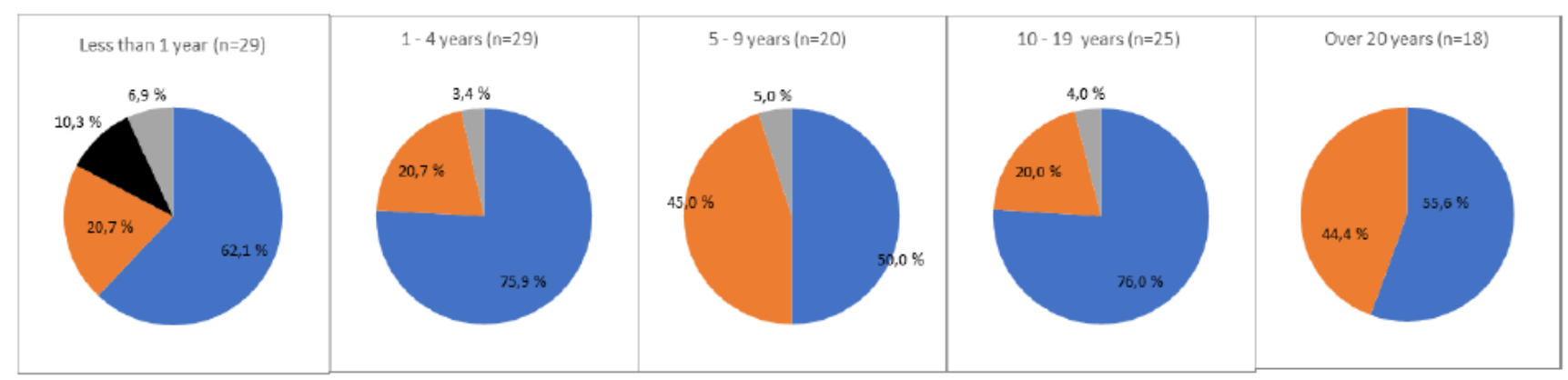

Figure 10. Reported Al utilization by healthcare professionals according to work experience. Blue, "Al can be used in all possible cases, but humans should always make final decision"; orange, "Al can ease clinical work and clinical processes but only with restricted matter (only by giving recommendations)"; black, "Al is not suitable for healthcare"; grey, "Al can be used in healthcare and can independently provide diagnoses and decisions for care".

solutions", and "AI does not provide accurate results for decision making. Only healthcare professionals can make right decisions". The disadvantages reported by the YouGov survey were "People need the human touch when it comes to their healthcare", "If something unexpected is found during surgery or in a test, I do not trust robots to make decisions on what to do", and "Only a human healthcare professional can make the right decisions". Therefore, a correlation in perceived disadvantages by both groups of respondents can be seen.

When analyzing the demographic data of respondents and survey responses, we concluded that age and staff group among healthcare professionals does not have significant effect on adoption of AI features or willingness to use healthcare services utilizing AI methods. There was also an indication from the survey results that respondents of a younger age and shorter work experience in the healthcare sector correlated negatively with willingness to adopt $\mathrm{AI}$ technology in healthcare.

In addition, we found that willingness to use novel features and services utilizing AI by healthcare professionals is high when novel features are used to help healthcare professionals in their decision making or work processes. Perceived disadvantages when using AI services mainly concerned loss of human interaction when robotics is used in healthcare and unwillingness to let AI make clinical decisions.

Overall, we conclude that using AI in healthcare is accepted by healthcare professionals and there are many AI enabled features and services where there could be of high potential in healthcare. However, developers and researchers of these novel AI services must keep in mind that the perceived disadvantages should be solved and human decision making and human interaction in these novel services is necessary, until these new services gain user acceptance and adoption in the healthcare sector. From the YouGov survey, these same acceptance criteria also apply when considering healthcare AI service adoption within the general public.

In our future research work we will evaluate more results of this survey to find the most effective combination of AI-services and technologies that could provide the best benefits for healthcare work processes, health outcomes, and preventive health processes. We will focus on providing services that could be easily adopted by healthcare professionals and the general public, and that should provide imminent enhancement in care processes. Moreover, we will provide a comparative study of healthcare technologies where $\mathrm{AI}$ is used as a key element and evaluate the effectiveness of these technologies in the field of healthcare. Finally, we will propose a technology platform where these studied AI technologies could be easily adopted by healthcare professionals and their customers or patients.

\section{Data availability}

Underlying data

Open Scientific Framework: Survey to healthcare professionals on the practicality of AI services for healthcare, https://doi.org/ 10.17605/OSF.IO/674ZM ${ }^{12}$.

\section{Extended data}

Open Scientific Framework: Survey to healthcare professionals on the practicality of AI services for healthcare, https://doi.org/ 10.17605/OSF.IO/674ZM ${ }^{12}$.

This project contains the following extended data:

- Survey questions in English and Finnish.

- Survey questions in table format (Tables 1-3)

Data are available under the terms of the Creative Commons Zero "No rights reserved" data waiver (CC0 1.0 Public domain dedication). 
1. Wisskirchen G, Biacabe BT, Bormann U, et al.: Artificial Intelligence and Robotics and Their Impact on the Workplace. IBA Global Employment Institute 2017; 2012-2017.

Reference Source

2. Bughin J: Artificial Intelligence the Next Digital Frontier. McKinsey Global Institute Discussion Paper. 2017.

Reference Source

3. Meskó B, Hetényi G, Györffy Z: Will Artificial Intelligence Solve the Human Resource Crisis in Healthcare? BMC Health Serv Res. 2018; 18(1): 545.

PubMed Abstract | Publisher Full Text | Free Full Text

4. McCall B: COVID-19 and Artificial Intelligence: Protecting Health-Care Workers and Curbing the Spread. Lancet Digit Health. 2020; 2(4): e166-e167. PubMed Abstract | Publisher Full Text | Free Full Text

5. Food and Drug Administration: Proposed Regulatory Framework for Modifications to Artificial Intelligence/Machine Learning (AI/ML)-Based Software as a Medical Device (SaMD)-Discussion Paper. 2019. Software as a Medica
Reference Source
6. PWC. Why Al and Robotics Will Define New Health. referred 12.2.2020. Reference Source

7. Davenport T, Kalakota R: The Potential for Artificial Intelligence in Healthcare. Future Healthc J. 2019; 6(2): 94-98.

PubMed Abstract | Publisher Full Text | Free Full Text

8. Reddy S, Fox J, Purohit MP: Artificial Intelligence-Enabled Healthcare Delivery. $J$ R Soc Med. 2019; 112(1): 22-28.

PubMed Abstract | Publisher Full Text | Free Full Text

9. ScienceDirect. Likert Scale. referred 20.3.2020.

Reference Source

10. SurveyCircle. 2019

Reference Source

11. Microsoft: Use the Analysis ToolPak to perform complex data analysis. referred 15.3.2020.

Reference Source

12. Väänänen A: Survey to healthcare professionals on the practicality of AI services for healthcare. 2020.

http://www.doi.org/10.17605/OSF.IO/674ZM 


\section{Open Peer Review}

\section{Current Peer Review Status: ? $\mathrm{X} \times$}

\section{Version 1}

Reviewer Report 19 April 2021

https://doi.org/10.5256/f1000research.26355.r81945

(c) 2021 Faggioni L. This is an open access peer review report distributed under the terms of the Creative Commons Attribution License, which permits unrestricted use, distribution, and reproduction in any medium, provided the original work is properly cited.

\section{Lorenzo Faggioni}

Diagnostic and Interventional Radiology, Department of Translational Research, University of Pisa, Pisa, Italy

This is an interesting manuscript reporting findings from a local survey on the expectations of healthcare professionals about the use of AI in healthcare. However, it has substantial flaws that should be addressed, including the following:

1. The survey findings and demographic data of the survey respondents were reported and illustrated in detail, but apparently without any sort of statistical analysis (which could allow drawing rigorous conclusions based on statistical significance). Hence, I would strongly encourage the authors to analyze their data using inferential statistical methods (including p-values, confidence intervals comparisons, etc.), in order to find objective associations and/or correlations in their findings. For instance, it would be very important to know if there were any statistically significant correlations between the replies of survey participants and their age, job/specialty and work experience.

2. The entire Discussion section should be focused on systematically comparing the study findings with those of the relevant existing literature. Basically, the only comparison was made with the YouGov survey, yet there are several surveys published in the literature regarding the awareness of healthcare professionals about AI and their expectations (either positive or negative) about its usability. Useful references include (but are not limited to) those listed in the References list attached to this review. 1,2,3,4,5,6,7,8,9,10,11

3. I would mention the rather small study sample ( $N=121$ complete responses) as a potential limitation of the study and comment on whether such limitation can impact the study findings and conclusions.

\section{References}

1. Yu KH, Beam AL, Kohane IS: Artificial intelligence in healthcare.Nat Biomed Eng. 2 (10): 719-731 PubMed Abstract | Publisher Full Text 
2. Coppola F, Faggioni L, Regge D, Giovagnoni A, et al.: Artificial intelligence: radiologists' expectations and opinions gleaned from a nationwide online survey.Radiol Med. 2021; 126 (1): $63-$ 71 PubMed Abstract | Publisher Full Text

3. European Society of Radiology ESR: Impact of artificial intelligence on radiology: a EuroAIM survey among members of the European Society of Radiology.Insights Imaging. 2019; 10 (1): 105 PubMed Abstract | Publisher Full Text

4. Eltorai AEM, Bratt AK, Guo HH: Thoracic Radiologists' Versus Computer Scientists' Perspectives on the Future of Artificial Intelligence in Radiology.J Thorac Imaging. 2020; 35 (4): 255-259 PubMed Abstract | Publisher Full Text

5. Krittanawong $\mathrm{C}$, Zhang $\mathrm{H}$, Wang $\mathrm{Z}$, Aydar $\mathrm{M}$, et al.: Artificial Intelligence in Precision Cardiovascular Medicine.J Am Coll Cardiol. 2017; 69 (21): 2657-2664 PubMed Abstract I Publisher Full Text

6. Patel UK, Anwar A, Saleem S, Malik P, et al.: Artificial intelligence as an emerging technology in the current care of neurological disorders.J Neurol. 2019. PubMed Abstract | Publisher Full Text 7. Pinto Dos Santos D, Giese D, Brodehl S, Chon SH, et al.: Medical students' attitude towards artificial intelligence: a multicentre survey.Eur Radiol. 2019; 29 (4): 1640-1646 PubMed Abstract | Publisher Full Text

8. Oh S, Kim J, Choi S, Lee H, et al.: Physician Confidence in Artificial Intelligence: An Online Mobile Survey. Journal of Medical Internet Research. 2019; 21 (3). Publisher Full Text

9. van Hoek J, Huber A, Leichtle A, Härmä K, et al.: A survey on the future of radiology among radiologists, medical students and surgeons: Students and surgeons tend to be more skeptical about artificial intelligence and radiologists may fear that other disciplines take over.Eur J Radiol. 2019; 121: 108742 PubMed Abstract | Publisher Full Text

10. Layard Horsfall H, Palmisciano P, Khan DZ, Muirhead W, et al.: Attitudes of the Surgical Team Toward Artificial Intelligence in Neurosurgery: International 2-Stage Cross-Sectional Survey.World Neurosurg. 2021; 146: e724-e730 PubMed Abstract | Publisher Full Text

11. Maassen O, Fritsch S, Palm J, Deffge S, et al.: Future Medical Artificial Intelligence Application Requirements and Expectations of Physicians in German University Hospitals: Web-Based Survey.J Med Internet Res. 2021; 23 (3): e26646 PubMed Abstract | Publisher Full Text

Is the work clearly and accurately presented and does it cite the current literature? Partly

Is the study design appropriate and is the work technically sound?

Partly

Are sufficient details of methods and analysis provided to allow replication by others? Partly

If applicable, is the statistical analysis and its interpretation appropriate? Not applicable

Are all the source data underlying the results available to ensure full reproducibility? Yes

Are the conclusions drawn adequately supported by the results? Partly 
Competing Interests: No competing interests were disclosed.

Reviewer Expertise: Artificial intelligence, Medical imaging, Radiology, Imaging informatics

I confirm that I have read this submission and believe that I have an appropriate level of expertise to state that I do not consider it to be of an acceptable scientific standard, for reasons outlined above.

Reviewer Report 01 April 2021

https://doi.org/10.5256/f1000research.26355.r81941

(c) 2021 Blease C. This is an open access peer review report distributed under the terms of the Creative Commons Attribution License, which permits unrestricted use, distribution, and reproduction in any medium, provided the original work is properly cited.

\section{Charlotte Blease}

General Medicine and Primary Care, Beth Israel Deaconess Medical Center, Harvard Medical School, Boston, MA, USA

Unfortunately, there are some serious problems with this survey.

The abstract does not tell us anything about the sample or response rate.

We later learn that the survey is drawn from a convenience sample drawn from a local network in Finland. There is therefore no assurance about the representativeness of health professionals in Finland. Indeed, the respondents include a variety of professions - physicians, nurses, admin staff, maintenance staff. Therefore we do not have a clear understanding of the attitudes/opinions about a particular demographic group of health professionals in Finland. The variety of perspectives limits the possibility of drawing robust inferences.

In the Introduction, there is no adequate literature review, nor a clear outline of the objectives in light of the background literature.

We also need some commentary on whether it is is useful to survey health professionals on these questions. i.e. why would we expect health professionals to have a clear understanding of the applications of AI/ML to healthcare? This was not addressed.

What do the investigators consider the utility of the results of the survey to be? Educational interventions?

The authors do not adequately address the limitations of the survey.

Is the work clearly and accurately presented and does it cite the current literature? Partly

Is the study design appropriate and is the work technically sound? No

Are sufficient details of methods and analysis provided to allow replication by others? No 
If applicable, is the statistical analysis and its interpretation appropriate?

I cannot comment. A qualified statistician is required.

Are all the source data underlying the results available to ensure full reproducibility? Partly

Are the conclusions drawn adequately supported by the results?

No

Competing Interests: No competing interests were disclosed.

Reviewer Expertise: Philosophy of medicine, informatics, future of the health professions

I confirm that I have read this submission and believe that I have an appropriate level of expertise to state that I do not consider it to be of an acceptable scientific standard, for reasons outlined above.

Reviewer Report 15 October 2020

https://doi.org/10.5256/f1000research.26355.r72922

(C) 2020 Chatterjee $\mathbf{P}$. This is an open access peer review report distributed under the terms of the Creative Commons Attribution License, which permits unrestricted use, distribution, and reproduction in any medium, provided the original work is properly cited.

\section{Parag Chatterjee}

${ }^{1}$ National Technological University, Buenos Aires, Argentina

2 Universidad de la República, Montevideo, Uruguay

The work is aimed at an interesting aspect of Artificial Intelligence in healthcare - the acceptability and opinions of the key stakeholders of the medical and healthcare system. The survey has been conducted in a well-structured manner, and the results are illustrated and analyzed well. However, considering the size of the cohort of this survey, a brief introduction and background of the participants would have been useful. Since some parts of the survey are quite subjective, it is important to explain the healthcare system and intrinsic relationships as well, where these insights from the survey hold true. The use of the words 'ICT Technology' is redundant, since ICT itself is 'Information and Communication Technology'. Based on the survey outcomes, it is interesting to see a high level of acceptance in healthcare professionals towards Artificial Intelligence in healthcare.

Is the work clearly and accurately presented and does it cite the current literature? Yes

Is the study design appropriate and is the work technically sound?

Yes 
Are sufficient details of methods and analysis provided to allow replication by others? Yes

If applicable, is the statistical analysis and its interpretation appropriate?

Partly

Are all the source data underlying the results available to ensure full reproducibility? Yes

Are the conclusions drawn adequately supported by the results?

Partly

Competing Interests: No competing interests were disclosed.

Reviewer Expertise: Artificial Intelligence, eHealth

I confirm that I have read this submission and believe that I have an appropriate level of expertise to confirm that it is of an acceptable scientific standard, however I have significant reservations, as outlined above.

The benefits of publishing with F1000Research:

- Your article is published within days, with no editorial bias

- You can publish traditional articles, null/negative results, case reports, data notes and more

- The peer review process is transparent and collaborative

- Your article is indexed in PubMed after passing peer review

- Dedicated customer support at every stage

For pre-submission enquiries, contact research@f1000.com

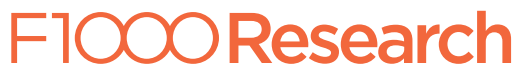

